\title{
Optical Spectroscopic Determination of the Zero-Field Splitting in Vibronic Levels of the Triplet State of Nitrite*
}

\author{
Robin M. Hochstrasser and Ahmed H. Zewail \\ The Department of Chemistry and Laboratory for Research on the Structure of Matter, The University of Pennsylvania, \\ Philadelplia, Pennsyliania 19104
}

(Received 19 October 1970)

\begin{abstract}
We have used conventional high resolution optical spectroscopic methods to determine the principal values of the fine structure tensor for the lowest triplet state $\left({ }^{3} B_{1}\right.$ at $\left.18959 \mathrm{~cm}^{-1}\right)$ of $\mathrm{NO}_{2}^{-}$in $\mathrm{Na} \mathrm{NO}_{2}$ single crystals. From the intensities in the Zeeman spectrum we find

$$
\begin{aligned}
& D-E=0.86 \pm 0.04 \mathrm{~cm}^{-1}, \\
& D+E=0.40 \pm 0.04 \mathrm{~cm}^{-1},
\end{aligned}
$$

where the $z$ axis is taken as the twofold axis and $E=(Z-X) / 2$. From the measurement of line positions in the high-field Zeeman spectrum we find $D-E=0.84 \pm 0.05 \mathrm{~cm}^{-1}, D+E=0.41 \pm 0.05 \mathrm{~cm}^{-1}$. The values of $D$ and $E$ calculated for one-center spin-spin interaction are $D-E=0.42 \mathrm{~cm}^{-1}$ and $D+E=0.24 \mathrm{~cm}^{-1}$. The calculated ordering of the spin states is the same as that observed, but we suggest that there is significant second-order spin-orbit energy associated with the real splittings. Roughly the same splittings are seen for different vibronic levels of ${ }^{3} B_{1}$ associated with $\nu_{2}{ }^{\prime}$.
\end{abstract}

\section{INTRODUCTION}

We wish to report on the measurement of the zerofield splitting of the triplet substates of various vibronic levels of the lowest excited triplet level of the nitrite ion $\left(\mathrm{NO}_{2}^{-}\right)$in sodium nitrite. $\mathrm{NO}_{2}^{-}$is an eighteen valence electron triatomic, isostructural with $\mathrm{O}_{3}$ and $\mathrm{SO}_{2}$. The zero-field splittings for triplet states of the neutral molecules can in principle be determined by rotational analysis, ${ }^{1}$ but a new approach is needed for ions: We present here two new approaches.

The lowest triplet state of $\mathrm{NO}_{2}-$ commencing at $18959 \mathrm{~cm}^{-1}$ was previously characterized as ${ }^{3} B_{1}$ by means of high field Zeeman spectra in polarized light ${ }^{2}$ and by pulsed Zeeman measurements. ${ }^{3}$ The ${ }^{3} B_{1} \leftarrow{ }^{-1} A_{1}$ transition is polarized along the molecular $y$ axis (the $y$-axis is in-plane, perpendicular to the principal $(z)$ axis; $\hat{y}$ is chosen to transform like $\left.B_{2}\right)$ and the $T_{z}\left(a_{2}\right.$ spin component) spin-state is considerably more radiatively active than $T_{x}$ or $T_{y}$.

Crystals of $\mathrm{NaNO}_{2}$ are orthorhombic Imm2.4 The $\mathrm{NO}_{2}{ }^{-}$site symmetry is $C_{2 v}$. All $\mathrm{NO}_{2}^{-}$molecules are equivalently oriented with respect to space fixed axes: The molecular $x, y$, and $z$ directions coincide with the crystallographic directions $a, b$, and $c$, respectively.

\section{THE EXPERIMENTS}

The first experiments consisted of precise measurements of the relative intensities of transitions to the low-field magnetic substates of the triplet level. The experiments are similar in principle to those previously presented ${ }^{8,9}$ but the method is especially suited to $\mathrm{NO}_{2}-$, and the results can be expected to be more accurate. Since predominantly the $T_{c}$ (or $T_{z}$ ) spinstate radiates, there are only very weak transitions to the $m_{s}=0$ state if a magnetic field of any strength is directed along the $a$ or $b$ axes: Thus a mainly two-line Zeeman spectrum is expected at all field strengths. The relative intensity of transitions to $m_{s}= \pm 1$ states for each field orientation $H_{\gamma}(\gamma=x, y$ or $z)$ provide the sign and magnitude of the zero-field splitting. It is readily shown $^{8}$ that the relative intensity is given by

$$
I_{+1} / I_{-1}=\left[1+\Omega\left(H_{\gamma}\right) / 1-\Omega\left(H_{\gamma}\right)\right]^{2},
$$

where the $\Omega\left(H_{\gamma}\right)$ are given by

$$
\Omega\left(H_{\gamma}\right)=\Delta E_{\gamma}{ }^{\mathrm{zf}} /\left(4 g \beta H_{0}-2 \Gamma\right)
$$

where $\Delta E_{\gamma}{ }^{2 f}$ is the separation between the zero-field states whose fine structure axes are perpendicular to the field; the indices are to be taken cyclically. $\Gamma$ is either $X, Y$, or $Z$ for $\gamma=x, y$, or $z$, respectively. These relations assume that $g$ is isotropic. A series of spectra for the vibronic band $0+\nu_{2}^{\prime}\left(\nu_{2}^{\prime}=644 \mathrm{~cm}^{-1}\right)$ as well as the 0,0 band are shown in Fig. 1 for both field orientations. More experimental details are given in the caption to Fig. 1. The other vibronic bands in the $\nu_{2}^{\prime}$ progression $(0 \rightarrow$ $n v_{2}^{\prime}$, where $n=2,3, \cdots, 6$ ) still exhibit the same general Zeeman splitting and intensity pattern for both field directions, but the lines get broad (thereby increasing the error to about $20 \%$ ) and we could detect no variation with vibronic state of the zero-field parameters. The results for 0,0 and 0,1 are

$$
\begin{aligned}
& D-E=0.86 \pm 0.04 \mathrm{~cm}^{-1}, \\
& D+E=0.40 \pm 0.04 \mathrm{~cm}^{-1} .
\end{aligned}
$$

The $T_{y}$ spin-state lies at lowest energy $(Y=-0.42$ $\left.\mathrm{cm}^{-1}\right)$ and $T_{x}$ lies at highest energy $\left(X=+0.44 \mathrm{~cm}^{-1}\right)$. The error of ca. $5-9 \%$ is a significant improvement over our previous work with less ideal crystals. ${ }^{8,9}$

A check on these indirectly measured fine-structure constants is available because the $T_{y}$ spin state is in 


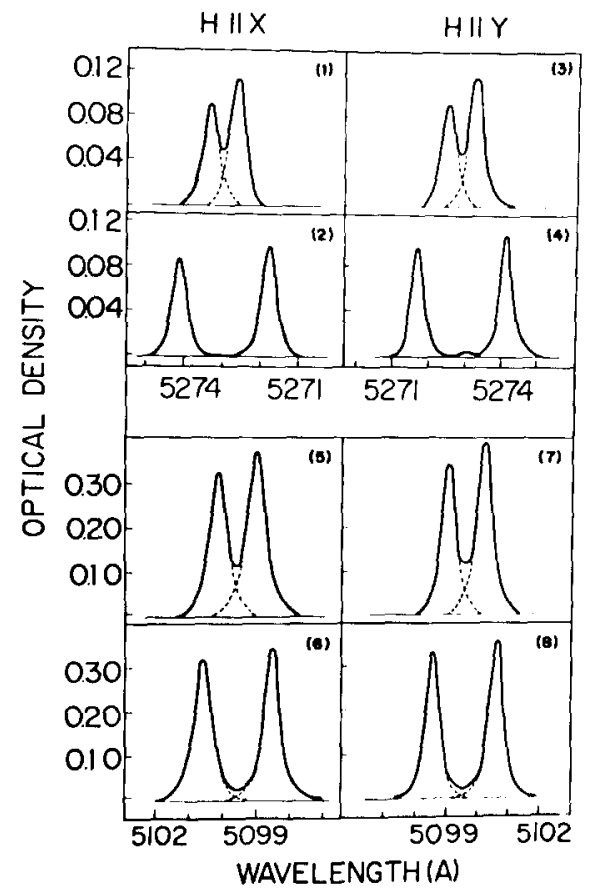

FIG. 1. Absorption spectra for 0,0 band (1-4) and $0,1\left(\nu_{2}{ }^{\prime}\right)$ band $(5-8)$ of $\mathrm{NO}_{2}^{-}$in $\mathrm{NaNO}_{2}$, at $4.2^{\circ} \mathrm{K}$. The 0,0 -band spectra are for $H=19.25 \mathrm{kG}(1,3)$ and $H=49.5 \mathrm{kG}(2,4)$. The 0,1 band spectra are for $H=27.5 \mathrm{kG}(5,7)$ and $H=49.5 \mathrm{kG}(6,8)$. The crystal is mounted with the $c$ axis perpendicular to $H$ and could be rotated about the $c$ axis. The spectra are all polarized parallel to the $b$ axis ( $y$ axis) of the crystal.

fact very weakly radiatively active. ${ }^{10}$ This means that with the field along the $y$ axis (b-crystal axis) a very weak center line is observed in the Zeeman pattern. This result is shown in Fig. 2. The optical resolution is $\approx 0.05 \mathrm{~cm}^{-1}$, and the value of $Y$ is given directly from the spectral line positions (without knowing the field strength!): We find $Y=-0.43 \mathrm{~cm}^{-1}$. The values of $X$ and $Y$ can also be obtained from the line positions and the value of $g \beta H$ : In that case we find $X=+0.43 \mathrm{~cm}^{-1}$, $Y=-0.42 \mathrm{~cm}^{-1}$. These values compare well with those obtained from the intensities, and indeed provide a confirmation of the general applicability of the indirect method.

\section{DISCUSSION}

In order to investigate the contributions to $D$ and $E$ from spin-spin interaction and spin-orbit interaction we have calculated the one-center contribution to the spin-spin interaction. The following four integrals were calculated using the Fourier convolution method, Slater-type orbitals and exponents ${ }^{11}\left(\mu_{2}\right)$ of 1.95 and 2.275 for $a=\mathrm{N}$ and $\mathrm{O}$, respectively:

$$
\begin{aligned}
A_{a}=\left(-\frac{1}{2} g^{2} \beta^{2} / h c\right) \iint 2 p_{x a}(1) 2 p_{y a}(2) & \left(r_{12}{ }^{2}-3 x_{12}{ }^{2} / r_{12}{ }^{5}\right) \\
\times & 2 p_{x a}(1) 2 p_{y a}(2) d \tau_{1} d \tau_{2}, \\
B_{a}=\left(-\frac{1}{2} g^{2} \beta^{2} / h c\right) \iint 2 s(1) 2 p_{x a}(2) & \left(r_{12}{ }^{2}-3 x_{12}{ }^{2} / r_{12}{ }^{5}\right) \\
\times & \times 2 s(1) 2 p_{x a}(2) d \tau_{1} d \tau_{2} .
\end{aligned}
$$

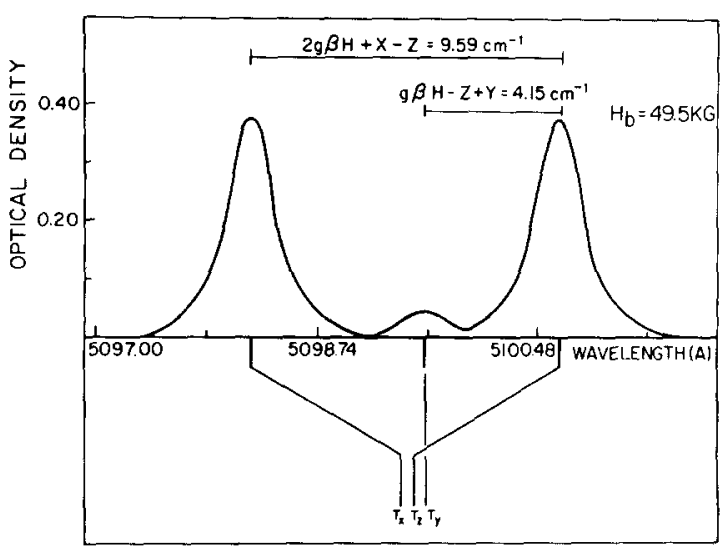

FIG. 2. High resolution absorption spectrum of 0,1 band. The crystal is mounted and the light is polarized as described in the caption to Fig. 1. The field is directed along the $b$ axis. One can clearly see the asymmetry of the Zeeman pattern, from which the fine-structure constants can be derived directly.

The zero-field splitting parameters can be expressed in terms of these four integrals: Using the calculated values $A_{\mathrm{N}}=0.44, A_{0}=0.69, B_{\mathrm{N}}=0.35, B_{0}=0.57 \mathrm{~cm}^{-1}$ we have obtained the following one-center contributions to the spin-spin interaction, based on the ${ }^{3} B_{1}$ wavefunction given by $\mathrm{McEwan}{ }^{12}$ :

$$
\begin{aligned}
& D^{(1)}-E^{(1)}=0.42 \mathrm{~cm}^{-1}, \\
& D^{(1)}+E^{(1)}=0.24 \mathrm{~cm}^{-1} .
\end{aligned}
$$

The simple one-center calculation gives the correct ordering of the spin-substates, but the fine structure constants are much too small. Perhaps the two-center spin-spin interaction would improve the agreement. We have tried including spin-orbit interaction in a simple way: If the total spin-orbit coupling energy of ${ }^{3} B_{1}$ with ${ }^{1} B_{2}$ states via $T_{z}$ was $\approx 35 \mathrm{~cm}^{-1}$, then the resulting interaction between ${ }^{3} B_{1}$ and ${ }^{3} B_{2}$ would shift the $T_{x}$ and $T_{4}$ states down by $\approx 0.2 \mathrm{~cm}^{-1}$. When this correction is applied, the calculated results become $D+E=0.44$ $\mathrm{cm}^{-1} D-E=0.82 \mathrm{~cm}^{-1}$ and these are encouragingly close to the experimental values considering that 35 $\mathrm{cm}^{-1}$ is about the spin-orbit coupling needed between ${ }^{3} B_{1}$ and ${ }^{1} B_{2}$ to explain the singlet-triplet absorption intensity. ${ }^{2}$ Spin-orbit interactions with ${ }^{3} A_{2}$ states, that would not be derivable from singlet--triplet absorption spectra because ${ }^{1} A_{2} \leftarrow^{1} A_{1}$ is forbidden, would lower the energies of $T_{y}$ and $T_{z}$. This is contrary to experiment if the one-center approximation for the spin-spin interaction is reliable.

* This research was*supported in part by a U.S. Department of Health Grant, G.M. 12592, and in part by the Advanced Research Projects Agency.

1 W. T. Raynes, J. Chem. Phys. 41, 3020 (1964).

${ }^{2}$ R. M. Hochstrasser and A. P. Marchetti, J. Chem. Phys. 50, $1727(1969)$.

${ }^{3}$ W. C. Allen and R. N. Dixon, Trans. Faraday Soc. 65, 1168 $(1969)$.

${ }^{4}$ S. Nomura, J. Phys. Soc. Japan 16, 2440 (1961). 
${ }^{5}$ G. E. Ziegler, Phys. Rev. 38, 1040 (1931).

${ }^{6}$ G. B. Carpenter, Acta Cryst. 5, 132 (1952)

7 M. R. Truter, Acta Cryst. 7, 73 (1954).

${ }^{8}$ R. M. Hochstrasser and T.-S. Lin, J. Chem. Phys. 49, 4929 (1968).

${ }_{9}^{9}$ R. M. Hochstrasser and T.-S. Lin, Symp. Faraday Soc. 3, $100(1969)$.

${ }^{10}$ With first-order spin-orbit coupling selection rules, the ${ }^{3} B_{1}$ state can connect with the ground state in dipole radiation via the $T_{z}$ spin state resulting in $\hat{y}$ polarization; or via the $T_{y}$ spin state resulting in $\hat{z}$ polarization. We showed previously ${ }^{2}$ that the former effect dominates. The observed transition to the $T_{y}$ spin state (with $H \| \boldsymbol{Y}$ ) is not obviously $\hat{z}$-polarized, and so its intensification mechanism is not certain at this time.

${ }^{11} \mu_{n}=Z_{n} / n$ where the principal quantum number $n=2: \mathrm{R}$. S. Mulliken, C. A. Rieke, D. Orloff, and H. Orloff, J. Chem. Phys. 17,1248 (1949).

i2 K. L. McEwan, J. Chem. Phys. 34, 547 (1961).

\title{
Kinetics of Diffusion-Controlled Reaction between Chemically Asymmetric Molecules.* I. General Theory
}

\author{
K. Šlolc† AND W. H. StockMaYer \\ Department of Chemistry, Dartmouth College, Hanover, New Hampshire 03755
}

(Received 26 October 1970)

\begin{abstract}
The treatment of diffusion-controlled bimolecular chemical reactions is extended to molecules with axially symmetrical distributions of reactivity on their spherical surfaces. Whereas an explicit solution cxists for the time-dependent effective rate constant at small times, the physically more interesting solution at large times can be obtained only approximately (though to any required degree of accuracy) by solving sets of linear equations.
\end{abstract}

\section{INTRODUCTION}

Much attention has been paid in the past to the kinetics of diffusion-controlled bimolecular reactions of spherical, chemically homogeneous molecules, the reactivities of which are the same all over their surfaces. A critical review of papers published until 1960 was given by Noyes ${ }^{1}$; some of the more recent papers ${ }^{2-6}$ take into account correlations among individual particles in the system ${ }^{4,5}$ and hydrodynamic interaction between pairs of diffusing particles. ${ }^{6}$ However, no attempt has been made, as far as we know, to analyze the effect of heterogeneity of surface reactivity. Although the assumption of uniform reactivity is sometimes justified, there are many cases (e.g., fluorescence quenching by highly asymmetric molecules, recombination of protein subunits, etc.) for which the reactive loci on the molecules are sharply specified and a steric factor should be considered. In this paper the Steinberg-Katchalski ${ }^{3}$ statistical method is generalized and applied to hydrodynamically spherical molecules with axially symmetric distributions of chemical reactivity. In a second paper, the results obtained will be compared with some simple approximate relations following from a quasichemical treatment of the diffusion.

We shall examine the kinetics of a diffusion-limited bimolecular irreversible reaction $A+B \rightarrow A B$. The molecules of both reactants are approximated as spheres of radii $r_{\mathrm{A}}$ and $r_{\mathrm{B}}$, and no interaction is assumed between them unless the distance $r$ between their centers is $r=a=r_{\mathrm{A}}+r_{\mathrm{B}}$. Since the reactive group represents in general only a small fraction of the total surface of the molecule, the surface should be described as chemically heterogeneous. A successful encounter resulting in chemical reaction requires not only sufficiently close approach of the two spheres but also their specific mutual orientation. If their position is such that at least one molecule is completely unreactive at the place of contact, no reaction can occur and the spheres may diffuse apart from each other. On the other hand, only if contact is made between strongly reactive spots on both spheres is success assured. In all intermediate cases a positive outcome of an encounter is only more or less probable. In many cases of practical interest the surface reactivity of $\mathrm{A}$ and $\mathrm{B}$ shows axial symmetry, and the distribution of the probability of reaction at a single encounter $W\left(\theta_{1}, \theta_{2}\right)$ can be described in terms of the polar angles $\theta_{1}$ and $\theta_{2}$, respectively, of the contact point with respect to the symmetry axes of both molecules. In this model, the state of each molecule is described sufficiently by giving the positional coordinates of its center and the orientation of the vector of its symmetry axis.

Following the approach of Steinberg and Katchalski, ${ }^{3}$ we shall estimate successively the probability of reaction between a single pair of $\mathrm{A}$ and $\mathrm{B}$, between an $\mathrm{A}$ and one of many surrounding B's, and finally the probability of reaction in the system composed of many A's and B's.

\section{PROBABILITY OF REACTION BETWEEN A SINGLE PAIR OF MOLECULES A AND B}

Starting from their initial positions, both molecules undergo Brownian motion characterized by two translational diffusion coefficients $D_{\mathrm{A}}, D_{\mathrm{B}}$ and two rotational diffusion coefficients $D_{\mathrm{A}}^{\text {rot }}, D_{\mathrm{B}}^{\text {rot }}$. Because translational and rotational motions are independent of each other 